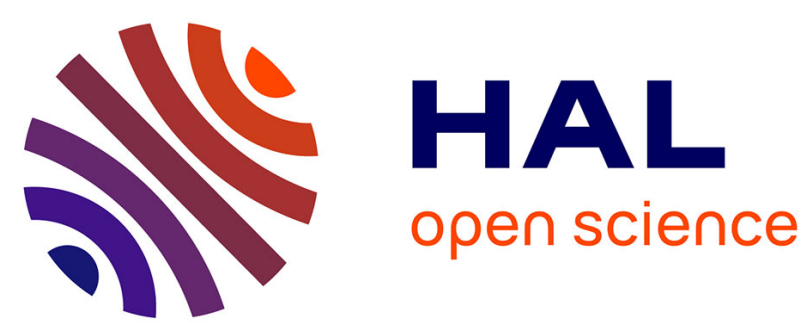

\title{
Les populations du Liban
}

\author{
Gérard-François Dumont
}

\section{To cite this version:}

Gérard-François Dumont. Les populations du Liban. Outre-terre. Revue européenne de géopolitique, 2006, 13, pp.419-445. 10.3917/oute.013.0419 . halshs-00769643

\section{HAL Id: halshs-00769643 https://shs.hal.science/halshs-00769643}

Submitted on 2 Jan 2013

HAL is a multi-disciplinary open access archive for the deposit and dissemination of scientific research documents, whether they are published or not. The documents may come from teaching and research institutions in France or abroad, or from public or private research centers.
L'archive ouverte pluridisciplinaire HAL, est destinée au dépôt et à la diffusion de documents scientifiques de niveau recherche, publiés ou non, émanant des établissements d'enseignement et de recherche français ou étrangers, des laboratoires publics ou privés. 


\title{
Les populations du Liban
}

\author{
Gérard-François Dumont
}

Après l'assassinat de l'ex-Premier ministre sunnite Rafic Hariri dans un attentat à la voiture piégée, le 14 février 2005, le peuple libanais a organisé d'immenses manifestations. Il a ainsi contraint la Syrie, qui occupait ce pays, à se conformer à la résolution 1559 du Conseil de sécurité des Nations unies et, par conséquent, à retirer ses militaires et, au moins officiellement, ses services associés au Liban ${ }^{1}$. La souveraineté du Liban devrait être restaurée, ce qui suppose de surmonter nombre de difficultés, compte tenu notamment du contexte géodémographique et géopolitique très particulier de ce pays.

Les spécificités de l'État libanais tiennent au caractère officiellement pluriconfessionnel de ce pays, avec un système de démocratie représentative le prenant en compte. Examinons d'abord le contexte géodémographique avant de nous interroger sur les éventuels traits unitaires justifiant le caractère national de cet État méditerranéen. Même si l'analyse conduit à plaider en faveur d'une véritable souveraineté libanaise, cette dernière se situe dans un contexte international et interne marqué par des constantes et des évolutions exerçant des effets sur les équilibres politiques libanais.

\section{Des traits géodémographiques singuliers}

Les traits géodémographiques du Liban tiennent d'abord à ses caractéristiques géographiques, explicatives de son originalité au Moyen-Orient comme

Gérard-François Dumont, professeur à l'Université de Paris-Sorbonne, président de la revue Population \& Avenir, 191, rue Saint-Jacques, 75005 Paris. Tél/fax \#33(0) 665744851. Gerard-Francois.Dumont@paris4.sorbonne.fr.

1. D'autres assassinats ont néanmoins eu lieu, comme celui de Samir Kassir, journaliste de renom et éditorialiste au quotidien al-Nahar, le jeudi 2 juin 2005. 
dans le monde arabe. S'y ajoute une dimension démographique fort modeste qui se caractérise par une densité remarquable et des diversités de peuplement selon les territoires.

\section{Une position géographique particulière}

Le Liban, qui fit partie de la Phénicie, connut dans l'Antiquité une civilisation brillante. Le Liban est un très ancien pays dont la prospérité est venue de sa position méditerranéenne, à l'interface des thalassocraties gréco-égéennes et des empires du Croissant fertile. À la fin du III ${ }^{e}$ millénaire, les Cananéens et les Phéniciens établissent sur la côte des comptoirs et fondent des cités-États (Tyr, Sidon, Byblos, Beyrouth). Les besoins du commerce nécessitant le recours à l'écriture, les Phéniciens créent un alphabet de vingt-deux lettres qui supplante l'écriture cunéiforme alors en usage et se répand dans toute la Méditerranée. Cet alphabet permet l'épanouissement de l'éducation, de la recherche et de la pensée. On peut constater que Babylone, Maris, Ninive ou Ur, qui furent de puissantes cités, ne sont plus que des tas de boue tandis que Tyr, reine du commerce entre l'Orient et l'Occident dès le $\mathrm{II}^{\mathrm{e}}$ millénaire avant J.-C., à environ $83 \mathrm{~km}$ au sud de Beyrouth, sous le nom de Sour, est la quatrième ville du Liban contemporain. Quant à Sidon, en dépit des vicissitudes de l'Histoire, elle est toujours vivante sous le nom de Saïda. La position géographique du Liban, tout comme celle de la Palestine qui prolonge le Liban au sud, explique le passage d'innombrables invasions qui ont laissé de fortes traces dans ces pays.

En 333 avant notre ère, avec la conquête d'Alexandre le Grand, l'indépendance de la Phénicie prend fin. À partir de cette période, et ce jusqu'au $\mathrm{XV}^{\mathrm{e}}$ siècle, le Liban disparaît en tant qu'entité politique souveraine ; il est englobé au sein d'une vaste zone dite "syrienne ». La domination grecque perdure d'abord pendant trois siècles, remplacée ensuite par la tutelle romaine. Puis, en 64 avant notre ère, les légions de Pompée fondent la Provincia Syria, choisissant Beyrouth comme métropole militaire et commerciale des Romains en Orient.

Dès la première moitié du $\mathrm{I}^{\mathrm{er}}$ siècle, le christianisme se propage dans cette province syrienne. En 395, lors du partage de l'Empire romain, la Provincia Syria, devenue chrétienne, est rattachée à l'Empire byzantin. L'Église maronite naît alors dans le diocèse d'Orient de l'Empire byzantin, constituant une branche de l'ancienne Église syriaque d'Antioche. En raison de sa position géographique, le Liban a toujours été au carrefour de trois continents et le passage obligé entre l'Occident et le monde arabe, bénéficiant au surplus d'un accès vers l'Asie centrale et la Russie. Par ce fait même, le pays fut maintes fois envahi et conquis par presque tous les peuples du bassin Méditerranéen, ou par des tribus nomades remontant de la péninsule Arabique. Enfin, du fait de sa 
configuration accidentée, ce pays montagneux a souvent accueilli les réfugiés de nombreuses contrées. Tous ces peuples ont laissé non seulement une empreinte architecturale, mais aussi un apport culturel et religieux important.

Aujourd'hui, l'État libanais est un petit rectangle d'orientation méridienne de $210 \mathrm{~km}$ sur 40 en moyenne (dans une fourchette entre 25 et 60 kilomètres), totalisant $10452 \mathrm{~km}^{2}$, soit le tiers de la Belgique ou l'équivalent de deux départements français moyens, mais moins que le département français métropolitain le plus vaste, la Gironde. Quelle est la population de ce territoire ?

\section{Un faible poids démographique}

Le poids démographique du Liban est modeste puisque, quelles que soient les sources, sa population varie entre 3,5 et 4,35 millions d'habitants. On peut être étonné par l'ampleur de cette fourchette, mais il convient de préciser qu'aucun recensement général n'a été effectué depuis 1932, sous le mandat français (1920-1946). Un fait qui s'explique peut-être par les implications éventuelles qu'auraient pu avoir ses résultats sur le partage politique du pouvoir.

Le nombre d'habitants ne fait donc l'objet que d'estimations assez différentes selon les sources. Le chiffre le plus bas est celui de la Division de la population des Nations unies, qui, dans le cadre de ses travaux, indique 3,577 millions d'habitants en 2005, la projection du Census bureau étant légèrement supérieure : 3,826 millions d'habitants. Mais ces chiffres sont nettement inférieurs à ceux qu'indique pour l'année précédente le Population Reference Bureau (PRB), soit 4,5 millions d'habitants ${ }^{2}$. Quant à l'Administration centrale de la statistique du Liban, elle ne fait figurer aucun chiffre sur son site web ${ }^{3}$. Néanmoins, selon l'enquête intitulée "Conditions de vie des ménages en $1997^{4}$ », le chiffre de 4,005 millions est avancé pour juin 1997, « avec un intervalle d'imprécision de plus ou moins 45771 dû aux aléas de l'échantillonnage ». Ce chiffre inclut une estimation des réfugiés palestiniens à 35000 . Toujours selon cette enquête, la population du Liban comporte environ 82,4 \% de Libanais, et donc 7,6 \% d'étrangers. La majorité des étrangers, soit 6,4\% de la population totale, serait constituée d'Arabes, répartis entre Palestiniens $(4,9 \%)$ et Syriens $(1,1 \%)$, sachant que les étrangers habitant temporairement la région, comme les ouvriers saisonniers (souvent syriens), ne sont pas comptabilisés. Le ministère des Affaires sociales indiquait pour l'année précédente, 1996, 3,4 millions d'habitants, dont 20000 à 30000 Palestiniens.

2. Cet organisme indiquant en revanche, pour 2005, 3,8 millions d'habitants, en retrait de 0,7 million par rapport à ses chiffres 2004 ! Cf. Population et Avenir, n 675, nov.-déc. 2005.

3. www.cas.gov.lb.

4. Études statistiques, nº 9, févr. 1998. 
Depuis ces chiffres officiels, difficilement comparables, de 1996 et 1997, la population pourrait avoir augmenté en raison du mouvement naturel, mais cette augmentation pourrait aussi avoir été limitée par un mouvement migratoire négatif. Il convient donc de s'accorder sur un chiffre estimatif, et nous proposons de retenir un chiffre moyen par rapport aux différentes estimations, soit 4 millions pour 2005 .

Si l'on retient ce chiffre, le Liban apparaît comme un pays peu peuplé dans le monde, dans le monde arabe comme au Moyen-Orient. En effet, sa population représente nettement moins qu'un pour mille de l'ensemble des populations du monde, estimé à 6396 millions en 2004.

Dans le monde arabe, sur 22 pays membres de la Ligue arabe, y compris les Territoires palestiniens, le Liban représente, en 2004, 1,42\% de la population des pays arabes, estimée à 317 millions d'habitants, et $0,07 \%$ de leurs 13,7 millions de $\mathrm{km}^{2}$.

Au Moyen-Orient, sur les 19 États concernés, y compris les Territoires palestiniens, le Liban représente, toujours en 2004, 1,3\% des 348 millions d'habitants et $0,1 \%$ des 7,35 millions de $\mathrm{km}^{2}$.

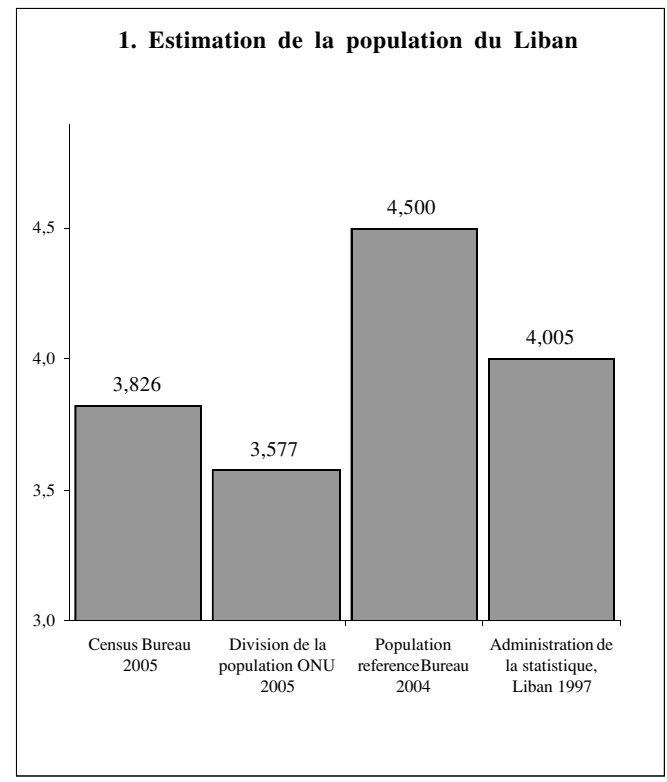

Si nous considérons à présent les pays proches ou limitrophes, le Liban apparaît comme l'État le moins peuplé. Concernant les pays limitrophes, Israël totalise 6,8 millions et la Syrie 18 millions d'habitants. Au-delà, les Territoires 
palestiniens ne comptent que 3,8 millions d'habitants selon les chiffres $2005 \mathrm{du}$ $\mathrm{PRB}$, mais beaucoup plus si l'on ajoute les réfugiés de Jordanie et du Liban. La Jordanie en compte 5,8 millions, y compris les réfugiés palestiniens ; l'Irak, 28,8 millions ; la Turquie, 71,9 millions. Ce faible poids démographique du Liban le place néanmoins en tête dans d'autres classements démographiques, comme la densité.

\section{Mais un peuplement dense...}

En effet, le Liban, malgré des possibilités de peuplement limitées ou rendues difficiles par le caractère très montagneux du territoire, compte une densité remarquable, entre 360 et 450 habitants par $\mathrm{km}^{2}$ selon les estimations de population. Une telle densité le place en $14^{\mathrm{e}}$ position ${ }^{5}$ de tous les pays du monde, mais en $9^{\mathrm{e}}$ position si l'on ne compte pas les cités-État comme Macao, Monaco, Singapour $\left(647 \mathrm{~km}^{2}\right)$, Hong Kong $\left(1068 \mathrm{~km}^{2}\right)$ ou Bahreïn $\left(690 \mathrm{~km}^{2}\right)$.

En ne considérant que les États de plus de $10000 \mathrm{~km}^{2}$, le Liban a la $4^{\mathrm{e}}$ densité du monde après le Bangladesh, Taiwan et la Corée du Sud, et devant les Pays-Bas (395) et la Belgique (340).

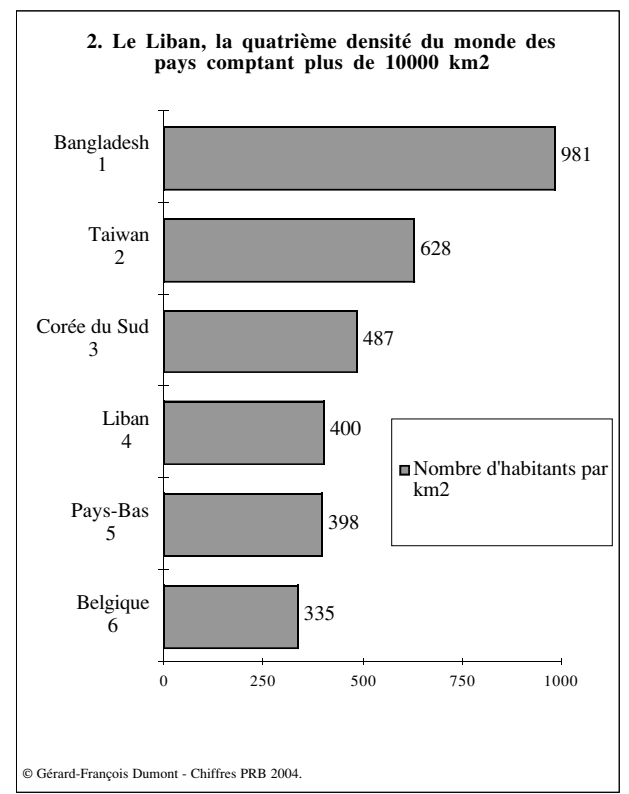

5. Cf. Gérard-François Dumont, Les Populations du monde (2 éd.), Paris, A. Colin, 2004. 
Une telle densité est également remarquable dans le monde arabe comme au Moyen-Orient, la densité moyenne des 22 pays de la Ligue arabe étant de 23 habitants $/ \mathrm{km}^{2}$ et celle des 19 pays du Moyen-Orient ${ }^{6}$ de 47 habitants $/ \mathrm{km}^{2}$.

Parmi les pays voisins, il faut noter la densité d'Israël (325 habitants $\left./ \mathrm{km}^{2}\right)$, qui compte $20620 \mathrm{~km}^{2}$, et, plus encore, des Territoires palestiniens (633 habitants $/ \mathrm{km}^{2}$ sur $6000 \mathrm{~km}^{2}$ ). Quant à la Syrie, sa densité est de 97 habitants $/ \mathrm{km}^{2}$.

Une densité moyenne nationale n'ayant qu'une signification limitée, il convient de préciser la répartition géographique de la population et l'armature urbaine du Liban.

\section{...et réparti sur le territoire}

Selon l'enquête de 1997, le poids démographique relatif de Beyrouth se serait abaissé de 22,3 \% de la population du Liban en 1970 à $10 \%$ en 1997. Mais cela doit être corrigé par l'essor démographique de la banlieue immédiate : la région métropolitaine de Beyrouth, additionnant Beyrouth et sa banlieue, héberge près du tiers de la population du Liban, soit 32,5\%. Cet attrait de l'aire urbaine de la capitale se confirme si l'on considère le lieu d'enregistrement : seulement un habitant de Beyrouth sur deux est originaire de cette ville, les autres venant des autres territoires libanais $(38,8 \%)$ ou de l'étranger $(10,4 \%)$.

Des six régions administratives, les muhafazats, la plus peuplée est le MontLiban, avec 37,6\% de la population du Liban, suivi du Liban-Nord (20,1\%) et de la Bekaa (13,6 \%). Le Liban-Sud, avec $11,8 \%$ de la population nationale, devance Beyrouth, tandis que le Nabatiyeh occupe la dernière position.

Un aspect de macrocéphalie peut être avancé en raison de l'importance démographique du grand Beyrouth (1,8 million dans la région métropolitaine de Beyrouth, dont 450000 dans la ville-centre) et du poids démographique nettement moindre des autres villes : Tripoli (200 000 habitants), Saïda (100 000) et Tyr $(70000)$.

Le contexte géodémographique général étant précisé, il convient d'examiner le caractère du Liban en se demandant dans quelle mesure ses populations peuvent ou non éprouver un sentiment national. Les analyses ci-après permettent de répondre positivement à cette question.

\section{Les facteurs d'unité}

Au regard de certains critères, les Libanais s'inscrivent dans une logique unitaire liée d'abord aux caractéristiques géographiques du territoire. S'ajoutent

6. Cf. notre carte du Moyen-Orient, in Population et Avenir, n 660, nov.-déc. 2002, p. 22. 
une homogénéité ethnique et linguistique et des histoires migratoires communes impliquant un souci de contacts avec l'étranger en raison du caractère méditerranéen précisé supra. Enfin, l'existence des « trente glorieuses » libanaises laisse en mémoire de brillantes possibilités de développement grâce à la qualité des hommes.

\section{Logique géographique}

Le Liban est d'abord une réalité géographique, comme son nom l'indique puisqu'il provient de la montagne Liban, culminant à 3090 mètres au Kornet el-Saouda. Ce pays méditerranéen présente une étroite plaine côtière dominée par le Mont-Liban, séparé de l'Anti-Liban par une haute plaine, la Bekaa. Cette haute plaine (aux environs de 900 mètres), située à l'est du Mont-Liban, est précieuse pour les productions vivrières des habitants de la montagne, ce qui explique différentes étapes politiques dans l'histoire de la région. Par exemple, après avoir obtenu de la France, dans les années 1920, la marche vers l'indépendance, les maronites demandent bien sûr que la Bekaa soit libanaise pour l'autonomie alimentaire des territoires montagneux, lesquels avaient souffert en 1916, pendant la guerre, du blocus turc.

À l'est de la Bekaa, le massif de l'Anti-Liban est un plateau désertique à environ 2300 mètres, dont la ligne de crête marque la frontière entre le Liban et la Syrie, avec un point culminant à 2814 mètres, au mont Hermon, sur la frontière.

À l'origine, l'homogénéité du territoire est donc géographique. Sa logique méditerranéenne, à laquelle est attaché un arrière-pays montagneux, peut faire songer, ceteris paribus, à celle des Alpes-Maritimes. Elle se complète d'une homogénéité ethnique et linguistique.

\section{Homogénéité ethnique et linguistique}

En effet, le peuplement du Liban comporte trois types de facteurs communs. D'abord, il est composé de populations sémites, terme qui s'applique à différents peuples, comme les Arabes et les Juifs, considérés comme provenant d'un groupe originaire d'Asie occidentale et parlant des langues apparentées.

Ensuite, le peuplement du Liban est pour l'essentiel arabe, en ce qu'il se rattache à cette langue sémitique à l'instar du chaldéen, de l'araméen ou de l'assyrien. Même si le Liban a connu temporairement, pendant des périodes de domination, des peuplements minoritaires européens ou ottomans, tous les Libanais sont arabes, à l'exception des Libanais arméniens ayant réussi à fuir le génocide turc.

Enfin, Le Liban est un pays très homogène sur le plan linguistique. L'arabe libanais appartient au groupe méridional, qui se différencie d'autres langues de 
l'Asie occidentale ayant une origine indo-européenne, comme le kurde ou l'arménien. Il comporte, bien entendu, de nombreuses formes dialectales. L'arabe est effectivement depuis 1943 la langue officielle, et la quasi-totalité des Libanais parle arabe ou l'une de ses variétés, même lorsqu'ils pratiquent d'autres langues comme le français ou l'anglais. L'article 11 de la Constitution du Liban précise : «L'arabe est la langue nationale officielle. Une loi spéciale déterminera les cas où il sera fait usage de la langue française. »

L'arabe parlé par la très grande majorité de la population est l'arabe levantin du Nord, appelé aussi « arabe libanais » ou « arabe syro-libanais ». Cependant, l'appellation « syro-libanais » pour désigner l'arabe libanais peut choquer certains Libanais en raison des relations politiques délicates avec la Syrie. Dans le monde, environ 15 millions de personnes parlent cet arabe levantin du Nord : outre les locuteurs du Liban, on en compte 8,8 millions en Syrie, 1 million en Argentine, 500000 en Turquie et 800000 dans d'autres pays (États-Unis, France, Australie, Canada...).

Les autres communautés linguistiques du Liban sont très minoritaires : leurs pratiques concernent l'arménien, le kurde kurmandj, l'arabe syrien, l'arabe égyptien, le chaldéen néo-araméen, le français, l'arabe irakien, l'espagnol, l'araméen, l'anglais, le grec, l'italien, le turc, le portugais et l'assyrien néo-araméen.

Au point de vue linguistique, l'arabe classique demeure donc la langue officielle du Liban, l'arabe libanais (ou levantin du Nord), la langue d'usage. En raison de l'histoire du Liban, le français et l'anglais, en plus de l'arabe classique, demeurent des langues relativement pratiquées comme secondes langues. L'utilisation du français reste courante, notamment au sein des communautés chrétiennes, surtout les maronites, alors que l'anglais est davantage privilégié par les musulmans et, en général, dans les échanges commerciaux. Les Arméniens et les Kurdes ont conservé leurs langues d'origine, mais parlent également le français (Arméniens) ou l'anglais (Kurdes). Étant donné que les Libanais ont toujours été de grands commerçants, la connaissance des langues étrangères est une pratique courante.

Un autre aspect unitaire du Liban tient à une histoire migratoire semblable pour la majeure partie de ses populations et à une tradition de liens extérieurs.

\section{Un peuplement né de multiples immigrations}

Les migrations passées expliquant le peuplement actuel du Liban s'inscrivent dans la longue durée. Dès le vII ${ }^{\mathrm{e}}$ siècle, les territoires montagneux offrent une terre d'accueil à des populations soucieuses de trouver un refuge. En 628, les Arabes envahissent la région et, après la défaite byzantine à la bataille de Yarmouk en 636, les villes de la côte libanaise tombent entre les mains des musulmans, qui placent la province sous leur autorité. Par suite de la conquête 
arabe, la population se modifie considérablement, car des Arabes, des Perses et des Juifs entrent dans le pays. Dès lors, les chrétiens côtoient les musulmans sunnites, et cette conquête musulmane de 636 réduit progressivement les chrétiens du Proche-Orient à une minorité. En revanche, les maronites réussissent à conserver une certaine autonomie en raison de leurs liens avec Byzance, avec l'Occident et avec les autres minorités du Mont-Liban. Mais des querelles religieuses déchirent les populations, et des oppositions se manifestent entre les différentes communautés confessionnelles.

La montagne sert alors de refuge, d'abord aux maronites (VIII ${ }^{\mathrm{e}}$ siècle), puis aux chiites ( $\mathrm{IX}^{\mathrm{e}}$ siècle), enfin aux druzes chassés d'Égypte ( $\mathrm{XI}^{\mathrm{e}}$ siècle). Après les deux siècles de domination franque, de 1090 à 1289 (capitulation de Tripoli), les autres villes libanaises passent sous l'autorité des mamelouks (milices arabes), qui gouvernent la région pendant deux siècles et demi, de la fin du XIII ${ }^{\mathrm{e}}$ siècle jusqu'à 1516, lors de l'arrivée des Ottomans. Pour fuir les mamelouks, de nombreux maronites cherchent asile dans la haute montagne libanaise (ainsi qu'à Chypre).

Au cours de la période ottomane, spécialement à partir du XVIII ${ }^{\mathrm{e}}$ siècle, le Liban accueille plusieurs minorités fuyant la persécution : des grecs et des syriaques catholiques y trouvent non seulement un gîte pour leurs communautés naissantes, mais des couvents pour leurs moines et un siège pour chacun de leurs patriarcats récemment créés.

À la fin de la domination ottomane, après 1915, le Liban redevient une terre d'accueil de minorités opprimées. Comme rappelé supra, quelques dizaines de milliers d'Arméniens fuyant la persécution turque s'établissent dans le pays, suivis par une immigration kurde (environ 60000 personnes). En 1921, d'autres Arméniens, devant évacuer la Cilicie occupée par la Turquie, arrivent avec les troupes françaises. Vers 1925, c'est l'arrivée de Kurdes qui fuient la répression turque tandis que, pendant cette période des années 1920 et 1930, viennent aussi se réfugier divers groupes de chrétiens orientaux.

Après la Seconde Guerre mondiale, en 1948, le Liban accueille 170000 Palestiniens chassés d'Israël qui s'installent dans des camps de réfugiés et fournissent la main-d'œuvre nécessaire aux « trente glorieuses » de prospérité économique. Cette arrivée massive est largement acceptée et apparaît tolérable dans un État à tradition pluriculturelle. Puis, suite à l'instauration dans les pays arabes des régimes, dits révolutionnaires ou socialistes, allant dans le sens d'une homogénéisation ethnico-religieuse, le Liban voit arriver de nouvelles vagues de minorités devenues indésirables dans leur propre pays : des assyriens, des syriaques et des chaldéens d'Irak, des alaouites de Syrie, des chrétiens d'Égypte... Après la guerre israélo-arabe de 1967 (guerre des Six Jours), d'autres réfugiés palestiniens affluent en masse. 
Au lendemain des affrontements de 1970 en Jordanie, l'Organisation de libération de la Palestine, chassée de ce pays, s'installe avec ses combattants sur le territoire libanais. Quelque 500000 Palestiniens s'établissent alors au Liban.

Ainsi, des premières communautés de réfugiés que sont les maronites, chrétiens qui refusent la conversion à l'islam, à partir du VIII ${ }^{\mathrm{e}}$ siècle, aux Palestiniens de 1970, contraints de quitter la Jordanie, le peuplement du Liban provient de multiples exodes. Le peuplement du Liban contemporain additionne donc diverses populations, très majoritairement arabes, ayant cherché des territoires leur assurant une certaine sécurité. Ce rôle de refuge explique l'importante densité de population dont nous avons noté supra qu'elle est l'une des plus fortes au monde.

Le Liban est donc composé de multiples confessions, dont dix-sept sont reconnues dans les institutions. Les douze confessions chrétiennes, dont la plus nombreuse regroupe les maronites, sont des Églises orientales, dont certaines sont rattachées à Rome (maronites, grecs catholiques, arméniens catholiques, syriaques catholiques, chaldéens catholiques), d'autres ne reconnaissant pas l'autorité du pape (grecs orthodoxes, arméniens orthodoxes, chaldéens orthodoxes, syriaques monophysites). S'ajoutent les catholiques latins et les protestants.

Les musulmans, plus nombreux depuis l'installation des Palestiniens, se partagent en sunnites et chiites, auxquels s'ajoutent des communautés syncrétiques, alaouites et druzes. En outre, il faut compter une très petite minorité juive. Enfin, en 1994, une nouvelle et dix-huitième communauté, les coptes, a été institutionnellement reconnue.

Pour consolider leur existence en dépit de leurs faibles effectifs et de leur caractère minoritaire, la plupart des confessions libanaises ont cherché au fil de l'Histoire des liens à l'étranger susceptibles de les conforter.

\section{Une logique de liens extérieurs}

En effet, quand une communauté estime ne pas avoir la place qui lui revient, ou quand elle se sent menacée, elle se met en quête d'appuis à l'extérieur. Ainsi les maronites réussirent-ils à conserver leur identité, malgré la conquête musulmane de 636, grâce au caractère montagneux du pays, mais aussi en raison de leurs relations avec Byzance, avec l'Occident et les autres minorités du MontLiban. Dès les croisades, puis à nouveau au XVI ${ }^{\mathrm{e}}$ siècle, l'Église maronite bénéficie de la protection de la France et sait s'allier avec l'Église catholique, s'assurant ainsi une autonomie et une pérennité la tenant à l'écart des multiples crises subies par les autres Églises orientales, tiraillées entre leur dogme d'origine et la chrétienté romaine. Plus tard, en 1860, la France intervient pour mettre fin au massacre de chrétiens par les druzes et impose en 1861 une entité autonome de droit international sur $6500 \mathrm{~km}^{2}$, le vilayet du Mont-Liban, territoire 
quasi-exclusivement bicommunautaire (maronite et druze), mettant fin à trois siècles d'une domination ottomane qui remontait à 1516. L'unification du MontLiban s'effectue alors sur la base du « gouvernement des druzes » depuis le Sud et de la démographie chrétienne à partir du Nord.

Cet exemple montre que la plupart des communautés libanaises parviennent au fil des siècles à maintenir leur existence par les liens entretenus dans d'autres pays. Les Libanais ne sont donc pas des autochtones enfermés dans leurs montagnes et les quelques terres qui les environnent, mais des populations ouvertes sur le monde pour des raisons géopolitiques, politiques, économiques ou religieuses. À l'unité de causalité du peuplement s'oppose une diversité de liens historiques ou diasporiques.

Un autre héritage commun des Libanais est cette période que j'ai appelée les « trente glorieuses », en utilisant la formule appliquée par Jean Fourastié à la France.

\section{Un pacte national unanimement accepté et fécondant trente glorieuses}

Lors du débonnaire mandat de la France, officialisé par la Société des Nations en 1920, l'arrêté n ${ }^{\circ} 60$ du Haut-Commissariat du 13 mars 1936 reconnaît les 17 communautés religieuses du Liban, et reconnaît à chacune le droit d'organiser sa juridiction et d'adopter sa législation de statut personnel. Puis l'indépendance se concrétise le 7 octobre 1943 par un pacte national dont l'objectif est de présenter un front uni face à la France, et par une Constitution dont l'article 7 établit une répartition, fondée sur le recensement de la population de 1932, des postes dirigeants entre les diverses communautés : le président de la République doit être maronite, le président du Conseil sunnite, celui de la Chambre, chiite. Quant à l'article 9 de la Constitution libanaise, il reconnaît aux différentes communautés religieuses une liberté totale de culte et leur accorde le droit de régir leur statut personnel par des lois particulières : "La liberté de conscience est absolue. En rendant hommage au Très-Haut, l'État respecte toutes les confessions et en garantit et protège le libre exercice, à condition qu'il ne soit pas porté atteinte à l'ordre public. Il garantit également aux populations, à quelque rite qu'elles appartiennent, le respect de leur statut personnel et de leurs intérêts religieux. »

Notons qu'il n'y a guère de mariages mixtes entre ces communautés, donc peu de relations familiales intercommunautaires. Les communautés se cantonnent souvent par village, ou par quartier dans les grandes villes.

Avant les événements postérieurs à 1974, la capacité du Liban à surmonter les risques de conflits intérieurs lui vaut d'être qualifié de "Suisse du MoyenOrient ». Sa prospérité économique, renforcée par les réseaux des diasporas libanaises en Europe, en Afrique et en Amérique, et même par les intérêts des 
pays pétroliers arabes qui apprécient les compétences financières libanaises, est réelle. La consolidation du jeune État libanais semble s'effectuer, malgré des convoitises syriennes manifestes.

Puis, en dépit de ces traits communs, la guerre civile de 1975-1989, suivie de la pax syriana, multiplie les difficultés. Il convient de comprendre les différentes évolutions internes et extérieures qui n'ont pas facilité le maintien de l'équilibre politique ni celui de la paix sociale.

\section{Les évolutions démographiques et l'équilibre politique}

Deux types d'éléments sont susceptibles d'expliquer les embarras du Liban. Les premiers sont plutôt de nature interne, les seconds liés au contexte géostratégique, politique, religieux et idéologique du Moyen-Orient.

\section{La question des différentiels démographiques}

L'élément interne qui complique l'équilibre politique libanais provient de la question de l'adaptation des normes initiales de répartition des pouvoirs selon les communautés religieuses à des évolutions démographiques différenciées. À l'origine, l'équilibre politique est défini avec précision. Par exemple, le régime électoral établi par la loi du 26 avril 1960 prévoit une répartition fixe des sièges au Parlement entre les communautés : sur 99 sièges, 20 doivent revenir aux maronites.

Or, des disparités démographiques entre les communautés, par rapport aux proportions antérieures, interviennent pour trois raisons : l'émigration comme l'immigration sont différenciées selon les confessions, ainsi que le mouvement naturel.

Même s'il est malaisé de donner des chiffres exhaustifs, faute de système d'information statistique suffisamment détaillé, diverses approches sont possibles. Rappelons d'abord la situation constatée lors du dernier recensement en 1932 : sur 630000 habitants $^{7}$, le Liban compte alors 200000 maronites (31\%), 43000 druzes, 125000 sunnites (19,8\%), 105000 chiites (16,6\%), 25000 étrangers et juifs, et 130000 autres chrétiens.

Examinons ensuite les flux migratoires et naturels contemporains.

7. Ce chiffre peut paraître faible, mais le recensement s'effectue avant le plein effet de la période de transition démographique. Cf. Gérard-François Dumont, Les Populations du monde, op. cit. 


\section{Des populations qui émigrent}

En fait, le système migratoire libanais n'est pas unidirectionnel. Il comporte aussi des vagues d'émigration. Dans la première moitié du XIX $X^{\mathrm{e}}$ siècle, une première vague se constate en direction des villes égyptiennes, flux qui se poursuit dans la première moitié du $\mathrm{XX}^{\mathrm{e}}$ siècle.

À partir de la seconde moitié du XIX ${ }^{\mathrm{e}}$ siècle, les massifs montagneux du Liban, qui étaient un espace migratoire de refuge, deviennent des terres d'émigrants cherchant ailleurs des conditions économiques meilleures. Le Liban enregistre une deuxième vague plus importante, essentiellement chrétienne, vers le continent américain. Selon diverses estimations, entre 1850 et 1950, de 400000 à 800000 Libanais quittent définitivement leur pays. Ce sont d'abord des paysans montagnards fuyant des conditions économiques très difficiles. S'y ajoute une petite élite intellectuelle qui part en Égypte et parfois aux États-Unis. Quant au pourcentage important de Libanais qui s'installent, dans la seconde moitié du XIX ${ }^{\mathrm{e}}$ siècle, en Amérique latine, ils sont désignés comme turcos (sujets ottomans).

À la fin du XIX ${ }^{\mathrm{e}}$ siècle, le Liban connaît une troisième vague d'émigration plus réduite, surtout chiite, dirigée vers l'Afrique noire française, de Dakar jusqu'en Afrique australe, où les Libanais prennent une place importante dans le commerce local. Plus tard, lors de la décolonisation des années 1960, la plupart des Libanais d'Afrique rentrent dans leur pays d'origine, favorisant ainsi l'émergence d'une classe moyenne au sein de la communauté chiite. De 1950 à 1974, les migrations, de moindre intensité, s'avèrent définitives pour le Canada et l'Australie, mais demeurent temporaires dans les pays arabes de la région du Golfe (Arabie saoudite et Émirats arabes unis).

À partir de 1975 et de la guerre civile, l'émigration a repris dans deux directions principales. La première concerne les pays du Golfe pour les plus qualifiés (Koweït, Émirats arabes unis...). La seconde vise l'Occident en général : ÉtatsUnis, Canada, France, Australie. En tant qu'ancienne puissance mandataire, la France reste un important pays d'accueil. Cette émigration d'une ampleur considérable concerne toutes les classes sociales, toutes les régions libanaises et les différentes confessions, mais plus particulièrement les maronites.

En 1991, la diaspora libanaise, dont l'évaluation est malaisée, est chiffrée à 2,5 millions par un spécialiste ${ }^{8}$. En 2005, certains l'estiment à environ 13 millions de ressortissants libanais, y compris leurs descendants, vivant à l'étranger, dont 10,7 millions en Amérique du Sud et en Amérique du Nord, 1,2 million en Afrique, 400000 en Europe, 400000 dans les pays arabes et 300000 en Australie.

8. Cf. Gérard Chaliand, Jean-Pierre Rageau, Atlas des diasporas, Paris, O. Jacob, 1991. 
Face à ces différentes émigrations, les immigrations les plus récentes, comme indiqué supra, concernent essentiellement des musulmans palestiniens.

\section{Les différentiels dans le mouvement naturel}

Aucune donnée ne livre des taux de natalité et de mortalité par confession. En revanche, grâce au décompte des naissances et des décès tenu par l'Administration centrale de la statistique du Liban, il est possible de mettre en évidence de forts différentiels régionaux correspondant à la répartition des confessions ${ }^{9}$. En effet, le sud des muhafazats du Liban-Sud et du Nabatiyeh est peuplé de chiites, alors que le nord de ces régions compte plutôt des maronites ou des druzes. La Bekaa est principalement habitée par des chiites et par des sunnites, hormis quelques territoires où vivent des maronites et des grecs catholiques. Le muhafazat Mont-Liban est essentiellement peuplé de maronites dans sa partie nord, mais sa moitié sud comprend toutes les variétés de confessions, notamment des druzes et des sunnites. Le muhafazat Liban-Nord est peuplé essentiellement de sunnites à Tripoli et au nord de Tripoli, de grecs orthodoxes et de maronites dans sa moitié sud, et de chiites à l'est, dans la frange proche de la Bekaa.

Tableau 1. Poids démographique relatif et nombre relatif de naissances dans les 6 muhafazats (régions ou gouvernorats) du Liban

\begin{tabular}{|l|c|c|}
\hline \multicolumn{1}{|c|}{ Muhafazat } & $\begin{array}{c}\text { \% de la population } \\
\text { en juin } 1997\end{array}$ & $\begin{array}{c}\% \text { des naissances } \\
\text { en } 2003\end{array}$ \\
\hline Beyrouth & $9,7 \%$ & $11,6 \%$ \\
\hline Mont-Liban & $38 \%$ & $20,1 \%$ \\
\hline Liban-Nord & $20 \%$ & $29,7 \%$ \\
\hline Bekaa & $13,6 \%$ & $7,6 \%$ \\
\hline Liban-Sud & $11,8 \%$ & $14,3 \%$ \\
\hline Nabatiyeh & $6,9 \%$ & $16,8 \%$ \\
\hline
\end{tabular}

(c) Gérard-François Dumont, chiffres Administration centrale de la statistique 1997 et 2004.

Pour estimer les taux d'accroissement naturel selon les régions, il convient d'abord de considérer la répartition régionale des naissances et des décès selon les six muhafazats (mot que l'on peut traduire par gouvernorat, région ou province).

9. Cf. Brigitte Dumortier, Atlas des religions, Paris, Autrement, 2002. 


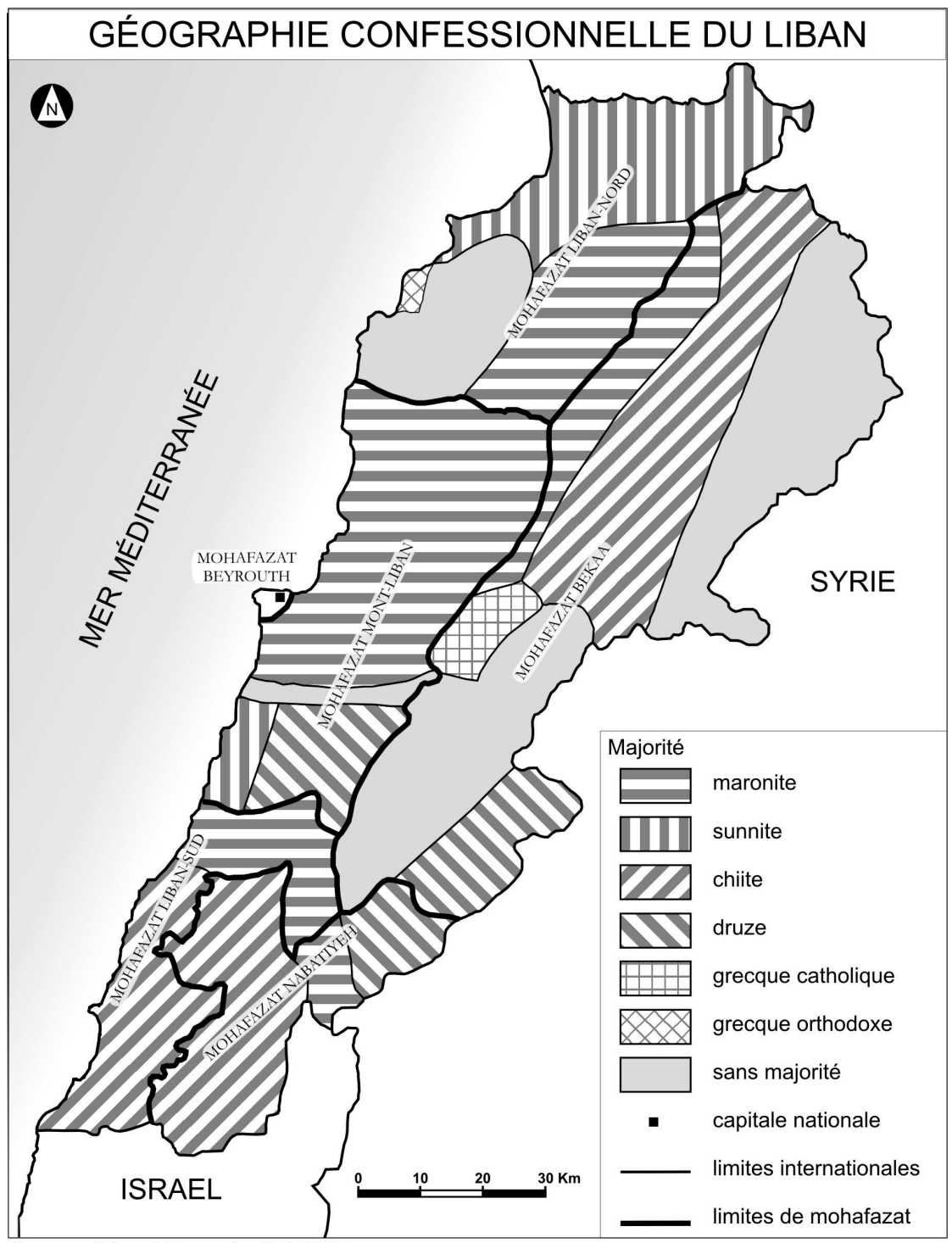

Source: Gérard-François DUMONT 


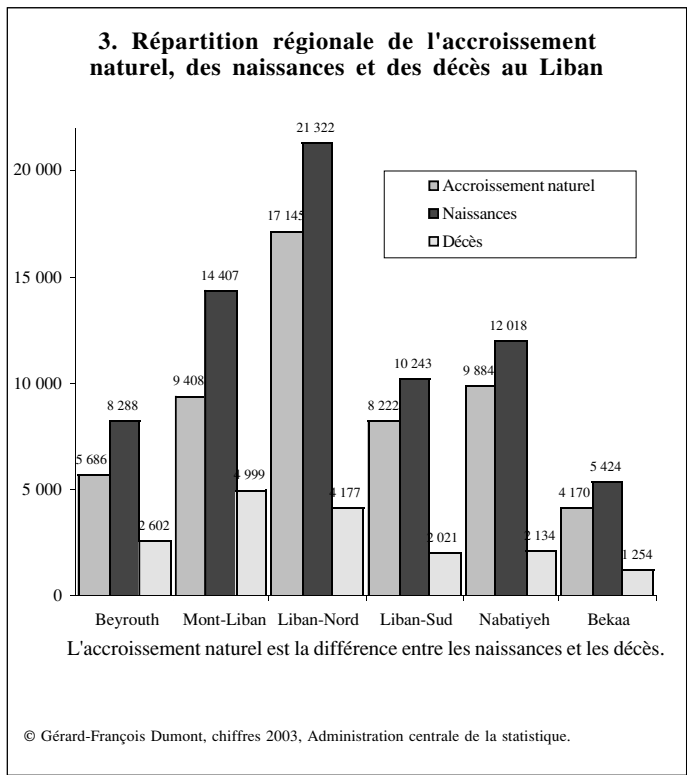

Le nombre de naissances le plus élevé s'observe au Liban-Nord, nettement devant le Mont-Liban, incluant la banlieue de Beyrouth, tandis que le chiffre le plus faible de naissances parmi les six régions se constate dans la Bekaa. En revanche, le nombre des décès est plus élevé au Mont-Liban qu'au Liban-Nord, ce qui s'explique par une composition par âge plus vieillie de la population.

En comparant la proportion régionale des naissances aux estimations de population de chaque région, on obtient des résultats fort variés. Deux régions semblent démographiquement dans une situation moins dynamique. Le MontLiban n'enregistre que 17,3\% de l'accroissement naturel pour $38 \%$ de la population. La Bekaa, muhafazat sans doute touché par l'émigration rurale et le vieillissement relatif qui en résulte, ne représente que 7,6\% de l'accroissement naturel annuel, avec 13,6 \% de la population. Tout cela traduit inévitablement d'importants écarts de fécondité, car, même dans l'hypothèse où la proportion de population du Mont-Liban et de la Bekaa serait surévaluée, il faudrait que cette surévaluation soit considérable pour justifier une fécondité proportionnellement plus élevée dans les quatre autres régions. Dans ces dernières, la part dans l'accroissement naturel est toujours supérieure au poids démographique relatif ; l'écart est faible à Beyrouth (10,4 \% de l'accroissement naturel national, 9,7 \% de la population du Liban). Elle est limitée au Liban-Sud (respectivement $11,8 \%$ et $15,1 \%$ ). En revanche, les écarts sont considérables au Liban- 
Nord (20\% et $31,5 \%$ ) et plus encore dans le muhafazat de Nabatiyeh : 6,9\% de la population, $18,1 \%$ de l'accroissement naturel. Un tel écart ne peut s'expliquer que par une fécondité nettement plus élevée, même si l'on peut penser que la population de cette région, au contraire de celle du Mont-Liban, est désormais sous-évaluée.

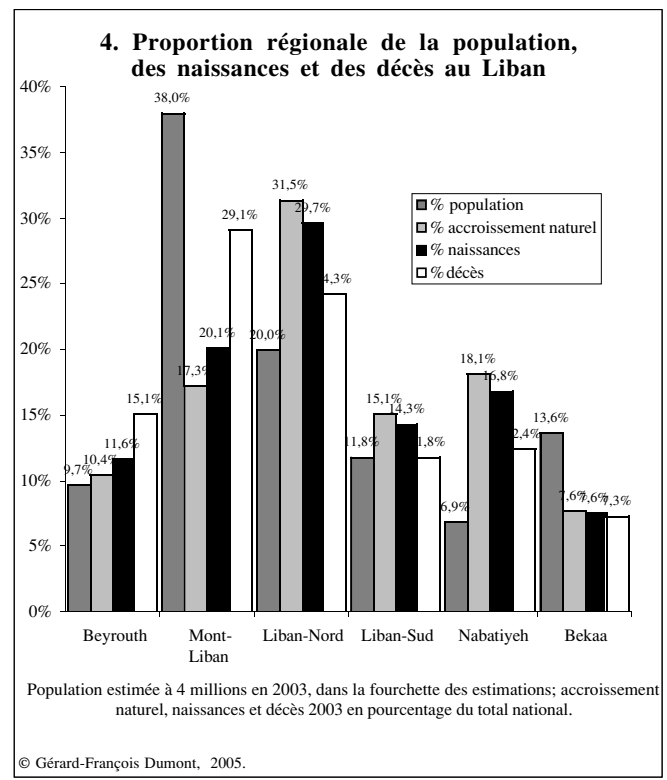

Ces différentes données permettent d'estimer des taux très disparates de natalité et de mortalité selon les régions. Conformément aux logiques mises en évidence supra, le taux de natalité du Mont-Liban et celui de la Bekaa seraient nettement inférieurs à la moyenne nationale. En revanche, le taux de natalité serait nettement supérieur, au double de la moyenne nationale, dans la région de Nabatiyeh, nettement supérieur dans le Liban-Nord et supérieur de quelques points à Beyrouth et dans le Liban-Sud.

Les niveaux différenciés des taux de mortalité ne sont guère aisés à interpréter ; le niveau inférieur à la moyenne nationale du Mont-Liban supposerait de connaître la distinction entre les taux de la banlieue de Beyrouth, à composition par âge plus jeune, et celui des territoires montagneux de cette région. Le faible taux de la Bekaa plaide plutôt pour une surévaluation de la population. Le taux le plus élevé, celui du Nabatiyeh, ne peut s'expliquer que par une sousévaluation de la population de cette région, ou par des taux de survie plus faibles 


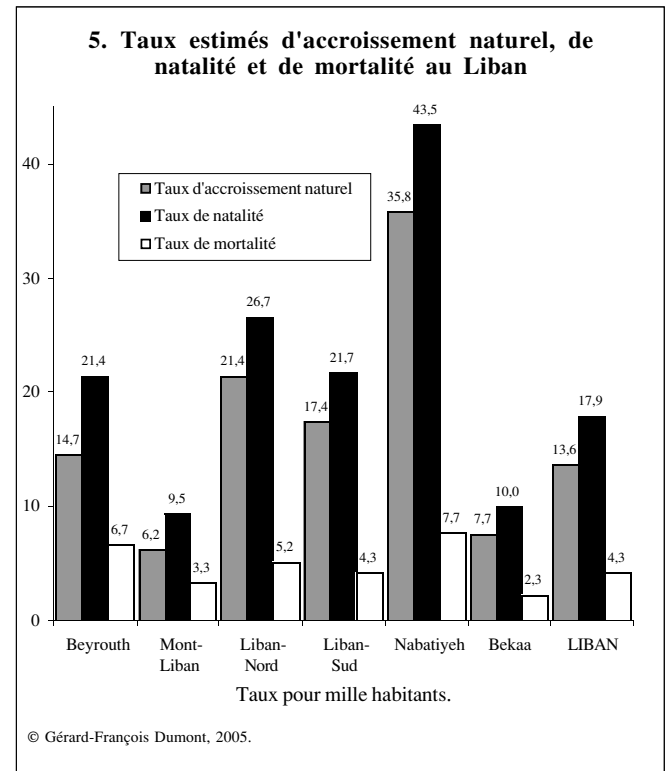

6. Répartition régionale des mariages et des divorces au Liban

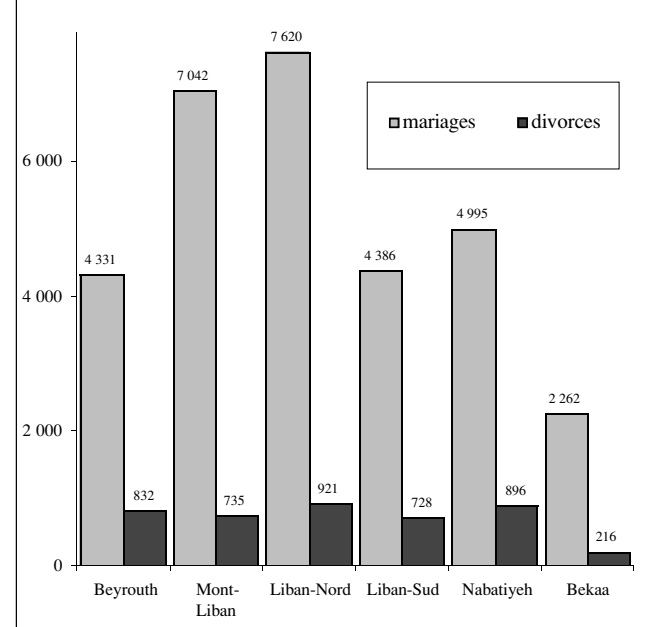

(C) Gérard-François Dumont, chiffres 2003, Administration centrale de la statistique. 
7. Proportion régionale des mariages et des divorces au Liban

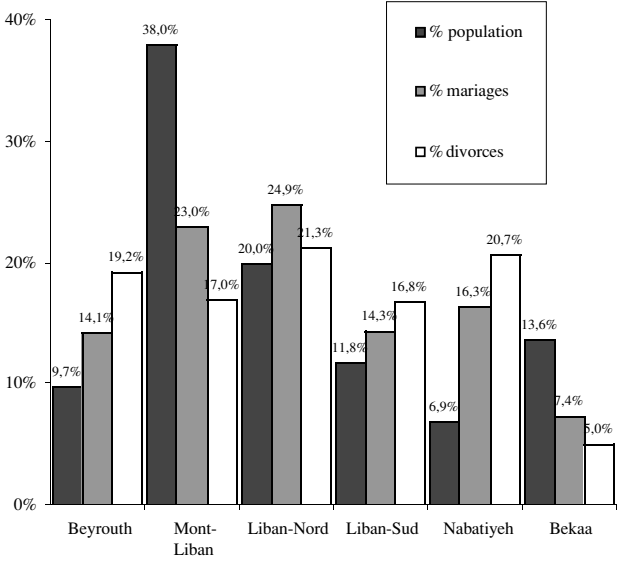

Population estimée à 4 millions en 2003, dans la fourchette des estimations; mariages et décès 2003 en pourcentage du total national.

(c) Gérard-François Dumont, 2005.

8. Estimation des taux de nuptialité et de divortialité au Liban

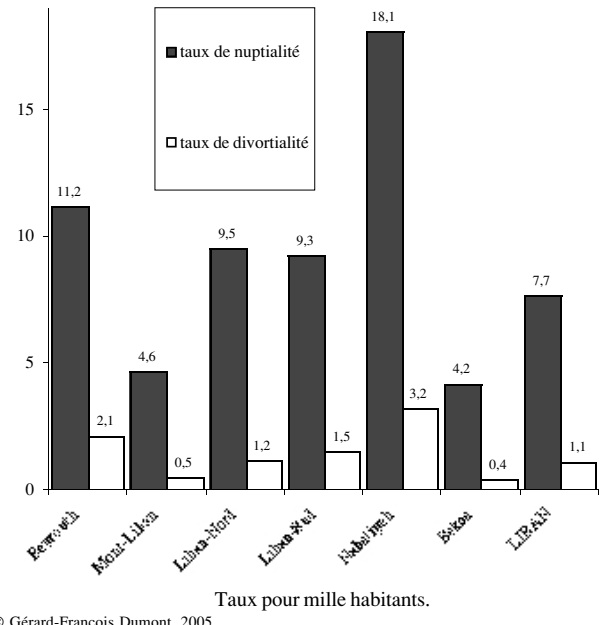


liés à la situation sanitaire et aux comportements d'hygiène. Quoi qu'il en soit, les écarts en faveur du Liban-Nord et du Nabatiyeh laissent à penser qu'existent des fécondités supérieures dans ces régions.

Si nous considérons désormais la nuptialité et la divortialité, les mariages les plus nombreux apparaissent dans les muhafazats Liban-Nord et Mont-Liban. En proportion de la totalité des mariages du Liban, le pourcentage des mariages est plus élevé que celui de la population au Liban-Nord, au Nabatiyeh, au LibanSud et à Beyrouth. En revanche, ce pourcentage est nettement plus faible au Mont-Liban, dont on peut penser que la population est relativement surévaluée, et dans la Bekaa, dont la population est peut-être plus vieillie.

L'estimation des taux de nuptialité indique des taux inférieurs à la moyenne nationale au Mont-Liban et dans la Bekaa, et le taux le plus élevé dans le Nabatiyeh, en raison de la structure par âge de la population et, sans doute, d'un âge au mariage assez jeune. Les taux de divortialité donnent également des écarts importants.

Toutes ces données sont évidemment complexes à traiter puisque l'attitude face au mariage est variable selon les confessions. Néanmoins, il est clair, malgré le caractère partiel des chiffres traités, que le Liban connaît de forts différentiels démographiques, avec des taux de croissance démographique plus élevés dans les territoires où l'on compte une proportion plus importante de musulmanes, chiites ou sunnites.

\section{Une estimation de la répartition confessionnelle}

La combinaison des différences du mouvement naturel et du mouvement migratoire selon les confessions permet de donner une estimation des confessions.

Il peut être acquis que les confessions chrétiennes, très légèrement majoritaires lors du recensement de 1932, ne le sont plus. Le poids démographique relatif de ces confessions chrétiennes aurait diminué d'une dizaine de points, d'environ $51 \%$ en 1932 à $40 \%$ en 2005. En revanche, le poids relatif des musulmans sunnites et chiites aurait augmenté, passant de 42,4\% en 1932 à $55 \%$ en 2005. Il serait donc attesté - premier enseignement - que le Liban de 2005 est majoritairement musulman.

Quant aux druzes, la proportion aurait logiquement baissé, de 6,8 \% à 5,0\%, puisque leur religion véhicule l'idée que la stationnarité démographique est nécessaire, le nombre d'élus ne devant pas se modifier.

Le second enseignement de l'estimation des confessions au Liban concerne les rapports entre les sunnites et les chiites, même s'il ne fait pas oublier que ces confessions ne sont pas totalement homogènes. Au recensement de 1932, la proportion des sunnites $(22,4 \%)$ est supérieure à celle des chiites $(19,6 \%)$. Or, 


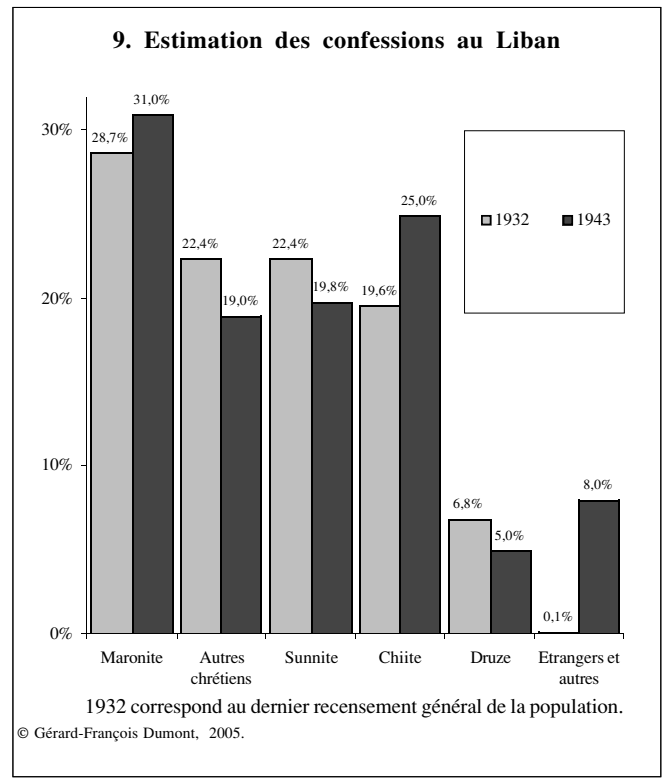

nombre d'éléments laissent à penser que cette hiérarchie se serait inversée au profit des chiites, qui pourraient désormais représenter $30 \%$ de la population, contre $25 \%$ pour les sunnites.

Ces changements dans la répartition de la population libanaise selon les confessions suscitent des interrogations. En effet, aucun texte ne prévoit une adaptation de la représentation politique aux évolutions démographiques, comme cela existe dans de nombreuses démocraties, à l'instar de la Chambre des représentants des États-Unis. Or, cette absence crée toujours nombre de difficultés. L'Union européenne elle-même a perdu et perd beaucoup de temps à négocier la représentation des populations dans ses instances institutionnelles. Elle continuera à en perdre puisque, à ce jour, aucun texte, y compris le projet de traité constitutionnel ${ }^{10}$, n'a remédié à cette question.

10. Notons que la question s'était déjà posée au sein de l'Union européenne lors de l'unification de l'Allemagne et qu'elle sera certainement posée dans le futur, puisque aucun traité ne la règle. Certes, le traité pour une constitution européenne signé à Rome le 29 octobre 2004 fixait un nombre minimum et un nombre maximum de parlementaires par État au Parlement européen, mais ce traité semble caduc depuis les votes négatifs de la France et des PaysBas des 29 mai et $1^{\text {er }}$ juin 2005. Quant aux chiffres des populations des États à retenir pour les décisions à la majorité qualifiée aux Conseils européens, ni ce traité ni celui de Nice ne précisent comment ils sont établis. Cf. Population et Avenir, mars-avr. 2005. 
La modification de la composition confessionnelle du Liban est donc de nature à créer des conflits internes, d'autant que ces derniers peuvent être attisés par un environnement géopolitique qui ne contribue guère à sa souveraineté et à sa stabilité.

\section{Un environnement géostratégique et idéologique peu propice}

La souveraineté du Liban heurte d'abord son grand voisin, la Syrie, qui ne l'a jamais admise. En outre, elle repose sur des principes antinomiques au contexte moyen-oriental.

\section{La Syrie et l'indépendance du Liban}

Il est impossible de comprendre l'attitude du peuple et du gouvernement syriens sans un bref retour en arrière ${ }^{11}$, au lendemain de la Première Guerre mondiale. Siège en 1920 d'un éphémère royaume hachémite auquel les Britanniques avaient fait miroiter le leadership d'un vaste empire arabe, Damas, l'orgueilleuse capitale Omeyyade, passe presque sans transition du joug ottoman au mandat français. Les Syriens sont alors choqués par la dislocation de leur pays en quatre États distincts (Alep, Damas, Alaouites et Djebel druze) à laquelle procède l'occupant français pendant une dizaine d'années. Dès 1920, le projet franco-maronite suscite une grande opposition en Syrie, qui n'a pas eu la possibilité de négocier sa frontière avec le Liban et craint d'être coupée de ses traditionnels débouchés vers la Méditerranée : Tripoli, Beyrouth et Saïda. Puis, en 1938, les Syriens sont traumatisés par la cession du territoire d'Alexandrette à la Turquie et par la colonisation sioniste en Palestine. La nouvelle entité territoriale libanaise n'est généralement pas acceptée par les nationalistes arabes, qui souhaitent dans leur ensemble la création d'une " Grande Syrie » englobant l'ensemble du Croissant fertile. Et la Syrie, devenue indépendante, se considère privée d'une grande partie de sa façade maritime sur la Méditerranée. De nombreux nationalistes syriens réagissent en revendiquant le Liban et même la Jordanie, qu'ils considèrent comme parties intégrantes de la " Grande Syrie ». Dans ce contexte, Damas n'a jamais établi de relations diplomatiques officielles avec le Liban. En 1961, la Syrie fait organiser un putsch par le Parti populaire syrien (PPS), mais celui-ci échoue.

La guerre civile, déclenchée le 13 avril 1975, est en un premier temps un conflit libano-palestinien sans coloration confessionnelle, opposant d'abord les

11. Cf. par exemple Marc Yared, «Le grand dessein de Hafez El-Assad », Les Cahiers de l'Orient, $\mathrm{n}^{\circ} 2,2^{\mathrm{e}}$ trim. 1986. 
phalanges chrétiennes aux Palestiniens, puis, à partir du mois d'août, aux milices islamo-progressistes alliées aux Palestiniens. En 1976, une intervention syrienne tente de contenir les Palestiniens. En 1978, Israël s'installe dans le Sud, avant de céder la place à une Force intérimaire des Nations unies au Liban (FINUL). Restant sur place, cette dernière ne peut empêcher la nouvelle intervention israélienne de 1982 (opération « Paix en Galilée »), au cours de laquelle Beyrouth est assiégée et l'OlP dispersée.

En 1983, l'armée syrienne intervient à nouveau contre l'OLP dans la partie ouest du pays (siège de Tripoli) pour empêcher cette organisation de faire du Liban une base arrière susceptible de transformer une partie du Liban en pays conquis par les Palestiniens. Elle contrôle alors $60 \%$ du territoire et occupe une position de force. La communauté internationale, craignant que les conflits entre communautés libanaises n'entraînent une rapide désagrégation du pays, semble préférer une pax syriana, installée par et pour les forces syriennes, à la continuation de la guerre civile. En 1989, les accords de Taëf permettent un retour progressif au calme, malgré une vaine résistance du général Michel Aoun, commandant l'armée chrétienne. En 1990, la prise de contrôle total du Liban par la Syrie s'effectue facilement, car le monde a une autre priorité : l'Irak a envahi le Koweït le 2 août 1990 et la Syrie se joint à la coalition internationale contre Saddam Hussein.

Dans cette période marquée par la guerre du Golfe, la Syrie et le Liban signent, en mai 1991, un «traité de fraternité et de coopération » qui revient à une reconnaissance officielle, au moins temporaire, de l'occupation du Liban par la Syrie.

Quinze ans après, le Liban d'aujourd'hui ne contrôle pas la totalité de son territoire et la souveraineté de l'État n'est toujours pas restaurée.

La question de l'attitude syrienne n'est pas la seule qui rende difficile l'équilibre politique du Liban. En effet, ce pays se trouve dans une situation atypique, par son caractère pluriconfessionnel, dans cet espace moyen-oriental où la tendance à l'uniformité religieuse apparaît comme un processus continu, au moins depuis le génocide arménien.

\section{La tendance régionale à l'uniformité religieuse}

Déjà, l'Arabie saoudite se considère non comme un territoire, mais comme une grande mosquée, ce qui la conduit à interdire non seulement tout lieu de culte non musulman, par exemple toute église chrétienne, mais même toute dose, si minime soit-elle, de liturgie d'une autre religion. En outre, l'évolution moyen-orientale se caractérise par un processus d'homogénéisation religieuse qui ne semble pas s'arrêter.

Ainsi, au début du $\mathrm{XX}^{\mathrm{e}}$ siècle, à la fin de l'Empire ottoman et après le génocide arménien, le Liban hérite de quelques milliers de rescapés. L'Anatolie, terre 
pluriconfessionnelle, renonce à cette qualité d'abord par une violence inouïe, puis par l'application du traité de Lausanne de 1923 qui déplace les orthodoxes résidant en Turquie et installe les musulmans qui vivaient auparavant en Grèce. Mais l'homogénéisation religieuse va au-delà de ce traité, car la Turquie ne respecte pas les droits des minorités qu'il prévoyait et poursuit sa purification ethnique par des moyens indirects, poussant les Turcs orthodoxes à l'exode. En 1971, le pouvoir turc ferme le seul séminaire orthodoxe existant encore, le collège théologique de Haiki, île proche d'Istanbul, et en interdit tout autre. Or, les douze métropolites qui élisent en synode le patriarche doivent tous être de nationalité turque et sortir de ce collège. Les Turcs de confession orthodoxe ne sont donc plus qu'une poignée, environ 3000 , même s'ils comptent parmi eux le patriarche œcuménique de Constantinople ${ }^{12}$.

Pour ne citer que quelques autres exemples du processus de refus de la pluralité religieuse au Moyen-Orient, en 1956, c'est Nasser qui pousse à l'émigration des Égyptiens juifs ou grecs, pourtant présents depuis près de deux millénaires. Ce sont aussi les attentats anticoptes, donc contre l'Église chrétienne la plus nombreuse de tout le Moyen-Orient, que l'Égypte connaît périodiquement. Certes, on voit des églises coptes en Égypte et, par exemple, celle qui se trouve à Assouan derrière l'hôtel Old Cataract où François Mitterrand aimait se rendre. Mais ces chrétiens d'Assouan sont eux aussi des réfugiés de l'intérieur, venus du Saïd, au nord. En Irak, les différents événements survenus depuis l'indépendance créent des vagues périodiques d'exode de chrétiens, quelle que soit leur communauté d'appartenance ${ }^{13}$.

L'histoire contemporaine de Chypre, pays que la géographie situe en Asie occidentale, est aussi celle d'une purification ethnique militairement contrainte en 1974, comme le symbolise la ville fantôme de Famagouste. De la même façon, les projets de création de deux États sur les terres de la Palestine géographique, par exemple celui de la feuille de route, organisent une séparation ethnique entre Arabes et Juifs, qui est aussi une purification religieuse même si demeurent des Arabes israéliens, d'autant que les Arabes chrétiens sont de plus en plus poussés à l'exode. Autant dire que le Liban se présente comme une anomalie face aux processus régionaux, ce qui lui rend difficile de préserver sa spécificité plurireligieuse ${ }^{14}$.

12. Cf. Gérard-François Dumont, «Populations et géopolitique en Turquie », Population et Avenir, $\mathrm{n}^{\circ}$ 670, nov.-déc. 2004.

13. Cf. Gérard-François Dumont, «La mosaïque des chrétiens d'Irak », Géostratégiques, $\mathrm{n}^{\circ} 6,2^{\mathrm{e}}$ trim. 2005.

14. Sur les religions, cf. Brigitte Dumortier, op. cit. ; Gérard-François Dumont, « Les religions dans le monde : géographie actuelle et perspectives pour 2050 », in Jacques Dupâquier, Yves-Marie Laulan, L'Avenir démographique des grandes religions, Paris, F.-X. de Guibert, 2005. 
Même la communauté internationale encourage souvent le processus moyen-oriental d'homogénéisation religieuse, dont les effets géopolitiques peuvent pourtant être graves dans la mesure où ils encouragent un esprit de fermeture, un repli sur soi, une difficulté à comprendre la différence et des risques de nationalisme. Par exemple, pendant la guerre du Liban, en 1976, les appels et les motions parcourent le monde, et humainement à juste titre, lorsqu'il s'agit de dénoncer le siège de Tall al-Za'atar, enclave palestinienne fortifiée implantée dans l'enclave chrétienne. Mais, à l'été 1978, personne n'élève la voix contre l'écrasement du quartier beyrouthin chrétien d'Achrafieh par les bombes syriennes. Il en est de même, en 1981, lors des bombardements de Zahlé, ville chrétienne assiégée depuis 1975 par des forces arabes étrangères au Liban. L'idée de purification religieuse est si répandue que les États-Unis vont émettre le souhait, en 1976, de transférer tous les chrétiens du Liban au Canada.

\section{Un contexte jacobin ou autoritaire}

Engagé pour préserver la cohabitation confessionnelle dans un contexte qui semble mal aimer la liberté religieuse, le Liban est également dans une situation difficile pour l'organisation de son État. Son caractère pluriconfessionnel explique, entre autres, la dénomination évoquée de «Suisse du Moyen-Orient », supposant en conséquence l'application du principe de subsidiarité et une « cantonisation » d'ailleurs souhaitable compte tenu du contexte géographique. La nature humaine du Liban suppose donc non un État jacobin, mais un État décentralisé ou fondé sur une forme de fédéralisme qui lui soit propre, sachant qu'il n'y a pas, dans l'histoire des institutions, une seule forme de fédéralisme, mais des formes liées à l'histoire et au génie de chaque peuple ayant choisi ce type de fonctionnement.

Or l'idée fédérale ${ }^{15}$, comme le principe de subsidiarité, n'est guère présente dans la région moyen-orientale. Les régimes politiques y sont plutôt jacobins ou autoritaires, que l'on considère la Syrie, la Turquie, l'Égypte, l'Iran ${ }^{16}$, l'Arabie saoudite ou la plupart des pays de cette Ligue arabe dont le Liban est membre fondateur. Le mode de fonctionnement de ces pays ne peut en rien servir de modèle ou d'inspiration institutionnelle au Liban, dont la réussite politique appelle, plus encore qu'ailleurs, l'application de principes subsidiaires.

15. Certes, en 2005, le mot «fédéralisme » apparaît dans la région au sujet de l'Irak. Mais guère en tant que fédéralisme subsidiaire, plutôt comme un mode de partage de la rente pétrolière, à l'instar de ce que l'on connaît au Nigeria.

16. Sur l'Iran, cf. Gérard-François Dumont, « La France et l'Iran : des nations si lointaines et si proches », Géostratégiques, n 9, 4 $4^{\mathrm{e}}$ trim. 2005. 
Atypique religieusement et politiquement au Moyen-Orient, le Liban subit en outre, et inévitablement, le contexte idéologique régional dont les évolutions apparaissent moins favorables à son idéal.

\section{Un changement de paradigme}

En effet, après la Seconde Guerre mondiale, l'idée de l'unité arabe, qui est le paradigme qui prévaut dans la région, favorise l'équilibre libanais. Une telle idée, qui aurait pu ou dû déboucher sur une sorte d'intégration s'inspirant de l'Union européenne ${ }^{17}$, est favorable à la nation libanaise. Le Liban étant arabe et de langue arabe, une telle intégration n'aurait pu que contribuer à son unité et à son développement. Ce sont d'ailleurs souvent des Arabes chrétiens qui ont illustré les premiers le nationalisme arabe. Une éventuelle intégration économique ne pouvait qu'être favorable au Liban, avec sa longue tradition d'ouverture ; elle aurait pu lui permettre de fonctionner en un pôle économique faisant bénéficier de sa culture et de ses compétences l'ensemble du Moyen-Orient.

Puis, pendant la guerre froide, le panarabisme s'est trouvé divisé par l'attitude de ceux qui voyaient dans un système politique et économique inspiré de l'empire soviétique l'avenir de la région, et de ceux qui devinaient que la voie soviétique, comme l'Histoire l'a montré, conduisait à une impasse dont on ne sort par définition qu'à reculons.

Ensuite, le paradigme de l'unité arabe est devenu moins prégnant avec la montée des islamistes radicaux ${ }^{18}$. L'arabité est d'autant moins un paradigme porteur d'avenir et facteur d'unité que le bilan de la Ligue arabe est terriblement négatif, même au regard des plus indulgents. Ce bilan désastreux a facilité la montée d'une autre idéologie, islamiste. Contrairement à l'arabité, qui se fonde sur l'ethnicité et n'interdit pas la pluralité religieuse, l'idéologie islamiste diffuse une vision manichéenne. Elle se fonde sur la haine des « juifs et des croisés », qui seraient responsables de tous les malheurs des musulmans, et leur associe tout musulman qui refuse de partager cette haine au nom de laquelle il est délivré un permis de tuer et même de se suicider, ce suicide interdit par la religion musulmane étant magnifié par les islamistes s'il résulte d'un acte

17. Cf. Gérard-François Dumont, «Unité européenne et unité du Moyen-Orient. Du paradigme nationaliste au paradigme du développement », Géostratégiques, n ${ }^{\circ} 8,3^{\mathrm{e}}$ trim. 2005. 18. Il faut bien entendu distinguer les « islamiques », c'est-à-dire les musulmans, des « islamistes », qui désignent en général une frange radicale. Les médias donnent souvent l'impression que cette question date du 11 septembre 2001. En réalité, elle est plus ancienne : cf. Jean-Pierre Péroncel-Hugoz, Le radeau de Mahomet, Paris, Lieu commun, 1983, ou Jean Bernières, «Terrorisme : pourquoi, qui et comment ? », Les Cahiers de l'Orient, $\mathrm{n}^{\circ} 3$, $3^{\mathrm{e}}$ trim. 1986. 
consistant à tuer des personnes qui ne participent pas de cette idéologie nihiliste. Un tel discours ne peut avoir que des effets déstabilisants sur un pays dont l'existence repose sur le refus de la haine entre les confessions qui le composent.

La diffusion du paradigme islamiste est telle que, par exemple, les médias présentent de plus en plus le conflit du Proche-Orient non comme un conflit entre Israéliens et Palestiniens, mais comme un conflit religieux entre juifs et musulmans. Pourtant les Palestiniens ne sont pas tous musulmans et ont longtemps inclus une importante minorité chrétienne, malheureusement progressivement écartée ou poussée à l'exode. Ainsi, en Palestine, en 1947, $20 \%$ de la population étaient chrétiens ; la proportion tombe en 1967 à $13 \%$. En 2005, dans les Territoires palestiniens, on ne compte plus que $2 \%$ de chrétiens et $6,7 \%$ pour la totalité des Palestiniens dans le monde ${ }^{19}$.

\section{Conclusion}

Pour tous ceux qui pensent que la diversité est créatrice, que toute purification ethnique et/ou religieuse ne peut conduire qu'à des réflexes de fermeture handicapant les possibilités de développement, pour ceux qui refusent le paradigme huntingtonien ${ }^{20}$, le Liban doit être considéré comme un diamant. En outre, le sentiment national qui s'est manifesté, de façon imprévisible et extraordinaire, après l'assassinat de Hariri le 14 février 2005 est une source d'espoir extraordinaire qu'il faut continuer de faire vivre. Une telle espérance doit être d'autant plus encouragée que le contexte contient néanmoins nombre d'éléments peu favorables à l'équilibre du Liban, qui est une mosaïque religieuse contestée, avec des frontières contestées et une souveraineté contestée.

Or, l'avenir du Liban passe désormais par la reconstruction d'une souveraineté dont la réussite suppose, d'une part, de marier le caractère pluriconfessionnel du pays avec certains éléments de laïcité et, d'autre part, un véritable soutien international pour contrecarrer les effets nocifs de facteurs déstabilisants.

19. Cf. Marwan Bishara, «Heurts et malheurs des chrétiens palestiniens », Le Figaro, 29 mars 2005.

20. Cf. Gérard-François Dumont, «Unité du genre humain et diversité des civilisations », Panoramiques, $\mathrm{n}^{\circ}$ 68, $3^{\mathrm{e}}$ trim. 2004. 